\title{
TIPO DE ORIENTAÇÃO CULTURAL E EMPATIA EM BRASILEIROS: VERIFICAÇÃO DE UM MODELO TEÓRICO
}

\author{
Nilton Formiga \\ Doutor em Psicologia Social pela Universidade Federal da Paraíba. Professor no curso de Psicologia \\ na Faculdade Mauricio de Nassau - João Pessoa-PB.
}

Marcos A. Souza

Doutor em Psicologia. Docente do Departamento de Psicologia e do Mestrado Profissional em Gestão e Estratégica em Negócios, na Universidade Federal Rural do Rio de Janeiro.

\begin{abstract}
Resumo
Os acontecimentos ocorridos na contemporaneidade têm afetado tanto os espaços sociais, econômicos e culturais, bem como as relações interpessoais. Acredita-se que uma orientação individualista ou coletivista influencia a capacidade das pessoas em reconhecer disponível para se colocar no lugar do outro: de ter empatia. O presente estudo tem como objetivo verificar um modelo teórico em que o tipo de orientação cultural explique a empatia. Participaram 456 sujeitos, do sexo masculino e do sexo feminino, de 12 a 67 anos, de instituições privadas e de públicas das cidades de João Pessoa-PB e Rio de Janeiro-RJ, que responderam a Escala Multidimensional de Reatividade Interpessoal, dos atributos do tipo de orientação cultural individualista e coletivista e dados sócio-demográficos. Observou-se uma associação positiva da orientação coletivista com a empatia, enquanto a orientação individualista associou-se, negativamente, com a empatia.
\end{abstract}

Palavras-chave: orientação cultural; empatia; modelagem estrutural.

\section{TYPE OF CULTURAL ORIENTATION AND EMPATHY IN BRAZILIANS: VERIFICATION OF A THEORETICAL MODEL}

\begin{abstract}
The events in contemporary have affected the social spaces, economic and cultural as well the interpersonal relations. It is believed that an individualist or collectivist orientation would influence people's ability to recognition of his capacity for interpersonal resonance: to empathize. The present study aims to verify a theoretical model in which the type of cultural orientation does associate with empathy. 456 subjects, male and female, ages from 12 to 67 years, of different educational levels of public and private institutions in the cities of Joao Pessoa-PB and Rio de Janeiro-RJ, answered to the Multidimensional Scale of Interpersonal Reactivity, the Scale of the Attributes of Individualistic and Collectivistic Cultural Orientation and sociodemographic data. There was a positive association between the collectivist orientation and empathy, on the other hand, individualistic orientation was associated negatively with empathy. It also highlighted the existence of a higher average score on collectivism which influences the high score on empathy.
\end{abstract}

Keywords: cultural orientation; empathy; structural modeling. 


\title{
TIPO DE ORIENTACIÓN CULTURAL Y EMPATÍA EN BRASILEÑOS: VERIFICACIÓN DE UN MODELO TEÓRICO
}

\begin{abstract}
Resumen
Los acontecimientos ocurridos en la contemporaneidad tiene afectado los espacios sociales, económicos y culturales, así como, las relaciones interpersonales; especialmente, en relación a la orientación que la persona tiene en la dinámica con el otro. Se cree que una orientación individualista o colectivista influencia la capacidad de las personas en reconocer disponible en ponerse en el lugar del otro, o sea, en tener empatía. El presente estudio tiene como objetivo verificar un modelo teórico en que el tipo de orientación cultural explique la empatía. 456 sujetos, del sexo masculino y del sexo femenino, de 12 a 67 años, de instituciones particulares y de públicas de las ciudades de João Pessoa-PB y Rio de Janeiro-RJ han respondido la Escala Multidimensional de Reactividad Interpersonal, de los atributos del tipo de orientación cultural individualista y colectivista y datos socio-demográficos. Se observó una asociación positiva de la orientación colectivista con la empatía, mientras que la orientación individualista se ha asociado, negativamente, con la empatía.
\end{abstract}

Palabras-clave: orientación cultural; empatía; modelación estructural.

\section{INTRODUÇÃO}

A dinâmica social, econômica e cultural, bem como, as relações interpessoais na contemporaneidade tem sofrido mudanças constantes: da linguagem, passando pela identidade social a política e tecnologia. Essas transformações têm afetado não apenas a parte concreta da sociedade, por exemplo, o mercado econômico e a urbanidade, mas também, as relações pessoais e sociais, as quais influenciam na apreensão de diferentes percepções no tipo e nas formas do conhecimento e experiências dos acontecimentos que ocorrem no entorno psicossocial. Isto tem modificado o processo do significado e sentido da vida indivíduo-sociedade em relação ao estabelecimento de um modelo de pensamento e conduta socialmente desejável, moral e ética (Jodelet, 1984; Howard, 2000).

Esse conjunto de mudanças, destacadas no parágrafo acima, mesmo que suscite melhorias na vida das pessoas e da sociedade tem levado especialistas das ciências humanas e sociais refletirem sobre a existência de uma sociedade que tem investido cada vez mais na experiência ambivalente, fluida, tendenciosa, constantemente, ao risco e com um foco na satisfação exacerbada para si mesmo (Burger, 1985; Kumar, 1997; Bauman, 1998; Lipovetsky \& Charles, 2004; Teixeira, 2005). Na literatura em geral sobre o desenvolvimento e história da civilização humana, é possível encontrar uma linha racionalistahistórica que percorre uma dinâmica da dicotomia em relação à orientação humana a individualidade-coletividade, a tradição-modernidade, o natural- 
adquirido, os quais, destinados ao estabelecimento de um padrão de conduta humana socialmente aceita. O fato é que, em termos sócio-históricos, geralmente, tende-se a priorizar uma ou outra dessas orientações objetivando compreender os processos psicossociais e a influência destes na manutenção de um tipo específico de conduta nas relações psicossociais. A análise de tal processo contribui para a explicação sobre o porquê e o como agir de forma cooperativa e com apoio social frente ao outro, principalmente (Barzun, 2002; Dumont, 1985; Elias, 1994; Fernández-Armesto, 1999).

Essas condições parecem influenciar a direção das mudanças culturais, justamente, que, por serem assim, podem alterar os modelos de pensamento e de conduta, fazendo com que mudem profundamente o entorno interpessoal, mas também, possa contribuir para identificar, na sociedade vigente, a existência dos conflitos entre o cultural e o legal, a conduta grupal e individual, a valoração pelo outro e a dissimulação cooperativa. Essa situação, que, dependendo da orientação adotada pelos sujeitos, leva pessoas e grupos à aderirem as condutas mais individualistas, uma vez que, segundo Formiga e Aguiar (2011) e Formiga (2012), as normas que governam o comportamento social estariam sem credibilidade ou se apresentando com uma maior valorização das coisas materiais, atitudes utilitaristas e auto-valoração de si mesmo, manifestando e deflagrando uma fissura na relação do cuidado e da valorização humana.

Frente à questão refletida no parágrafo anterior, o fator determinante é que, aqueles indivíduos que priorizam e continuam a ter na sociedade e nas normas sociais que a governam, uma segurança ainda mantém uma postura coletivista, indicando uma crença nos antigos ideais da sociedade, entendida como uma grande família, pronta a cuidar das pessoas que dela fazem parte; o contrário, a priorização de si mesmo e da mudança social e normativa para atender seus próprios objetivos, mesmo que prejudique uma dimensão mais ampla (isto é, a sociedade) revela a postura de um sujeito individualista. Este último tem sido a grande investida nas imagens da interpessoalidade que a sociedade contemporânea tem salientado (Souza, 2003; Formiga).

De acordo com Atteslander (1999), se o comportamento das pessoas depende de como elas percebem a situação em que se encontram, é possível que, a partir dessa apercepção, elas possam definir sua própria realidade e, consecutivamente, sua relação social e interpessoal com os outros; sendo assim, 
essa condição, influenciaria principalmente, na orientação cultural apreendida por elas, causando disposição das mesmas para as trocas de experiências, cognitiva e afetiva, em relação ao outro. Isto é, considerando as orientações culturas seguidas na atualidade, as quais visam um amplo individualismo e uma espécie de dramaturgia social e narcísica (Dumont, 1985; Lipovetsky, 1986; Lyotard, 2000), acredita-se que tal orientação possa influenciar o desenvolvimento do sujeito em relação ao reconhecimento de sua capacidade de ressonância interpessoal em direção de uma ética e moralidade relacional.

De acordo com Depraz (2005), tal ética, tem o foco no respeito e compreensão do outro e a inclusão da pessoa no campo do problema do outro, a fim de promover uma atitude de abertura psicossocial ao binômio problemapessoa. Neste sentido, conduz o psiquismo humano, tanto de quem precisa de ajuda quanto daquele que ajuda (ou melhor, de quem se empatiza) a uma disposição ao acolhimento dos outros e o desdobramento de horizontes de sentido da situação sofrida, reabrindo espaço para interação humana e seus vínculos afetivos.

\section{A perspectiva dos atributos de orientação cultural individualista e coletivista}

Ao enfatizar a respeito dos padrões convencionais da orientação cultural adotada por cada pessoa, destaca-se o papel dos atributos dos valores culturais proposto por Triandis (1995; 1996), distribuídos em valores de individualismo e coletivismo. Rokeach $(1973 ; 1979)$ já considerava esse construto fundamental quando se tratava de explicar os comportamentos das pessoas, o qual seria capaz de orientar tanto as escolhas quanto as atitudes humanas.

De fato, seja na mídia, transeuntes ou especialistas da ciência humana e social, percebe-se que uma preocupação frente à conduta social juvenil tem sido referida à cultura do indivíduo, estigmatizando-a como a única 'CULTURA' e destacando-a uma condição sine quo non do desenvolvimento sócio-humano dos jovens, excluindo a complexidade e diversidade humana frente à gestão e formação da conduta socialmente desejável nas relações interpessoais, fundamentada na assimilação e orientação de atributos culturais coletivistas e individualistas (Formiga, 2004; Formiga \& Diniz, 2011).

Neste contexto, ao considerar estes atributos como padrões de orientação cultural permitem ao sujeito a adoção de um ou outro tipo de orientação, o indivíduo irá se comportar de forma coerente com este. Por um lado, o 
individualismo expressa uma tendência ao sucesso, a valorizar a própria intimidade e uma necessidade de adequar-se ao contexto social, visando obter recompensas; por outro, o coletivismo define uma tendência à cooperação e ao cumprimento com relação aos demais; internamente, as pessoas com orientação coletivista, mantêm fortes relações entre si, podendo compartilhar os mesmos interesses (Gouveia, Clemente \& Vidal, 1998).

Desta forma, o individualismo e coletivismo são definidos como síndromes culturais, consistem em compartilhar atitudes, crenças, normas, papeis sociais e definições do eu, sendo os valores dos membros de cada cultura organizados coerentemente sob um tema (Triandis, 1995; 1996). As pessoas que se orientam por um tipo ou outro de orientação cultural, vão se comportar de maneira diferente, seja na forma de se auto-perceber ou nos seus relacionamentos interpessoais.

No entanto, deve-se salientar que o individualismo e coletivismo não são necessariamente opostos. Como assinalam Sinha e Tripathi (1994), as pessoas são um pouco de cada um, sendo o contexto ou a situação imediata, que vai definir o estilo mais apropriado de comportamento - o tipo de orientação (Triandis, 1995; Triandis, Chen \& Chan, 1998). Em todo caso, espera-se que, em cada pessoa, predomine uma destas orientações, não se podendo ignorar a possibilidade de coexistência das duas (Schwartz, 1990; Sinhá \& Tripathi, 1994), bem como, sua relação entre esses atributos.

Triandis (1995), ao recuperar a clássica dimensão de poder proposta por Hofstede (1980) que avaliava as dimensões culturais nas empresas, enfatizando o papel do sujeito ou do grupo na dinâmica das sociedades humanas, identifica dois atributos chave para diferenciar os principais tipos de individualismo e coletivismo: horizontal e vertical. O atributo horizontal sugere que as pessoas são similares na maioria dos aspectos, especialmente no status. O conceito vertical põe ênfase em aceitar a desigualdade e privilegiar a hierarquia. Estes atributos se combinam com o individualismo e coletivismo formando quatro tipos de orientação, cada um com uma característica principal que melhor descreve a pessoa que adota cada um destes tipos, a saber: 
- Individualismo $\left\{\begin{array}{l}\text { Horizontal - Ser único, diferente dos demais } \\ \text { Vertical - Orientado ao êxito, ao triunfo }\end{array}\right.$

- Coletivismo $\left\{\begin{array}{l}\text { Horizontal-Cooperador, que colabora } \\ \text { Vertical - Cumpridor com os demais, ser servidor }\end{array}\right.$

Considerar estes atributos - do individualismo e do coletivismo - permite relacionar uma variedade de fatos e pensamentos nas múltiplas facetas da vida social e política (Inglehart, 1991). Sabendo-se ainda que quando se deseja explicar comportamentos deve-se recorrer às atitudes, ao reconhecer a natureza atitudinal que estes construtos venham apresentar, é possível que eles contribuam para a explicação de construtos psicológicos (comportamentos, atitudes, etc.) (Rokeach, 1973; 1979; Formiga, 2004; Formiga \& Diniz, 2011); no caso do presente estudo, o construto da empatia em jovens.

O construto da empatia: O outro como um Ser significativamente importante

A empatia refere-se a uma disposição funcional das pessoas para as trocas de experiências expostas, incondicionalmente, em relação ao outro (Wispé, 1990; Decety; Jackson, 2004; Decety, 2005; Enz \& Zoll, 2006; Decety, Michalska \& Akitsuki, 2008). Esse construto pode ser definido como uma resposta afetiva de origem evolutiva que é mais apropriada à situação do outro do que da própria pessoa.

Com isso, uma pessoa empática, teoricamente, é capaz de experimentar vicariamente emoções sentidas por outra pessoa, adotar o ponto de vista do outro, compreender suas motivações e necessidades e atribuir atitudes e comportamentos ao outro com a função de prover ajuda, agregação, cuidado, justiça e solidariedade (Mehrabian \& Epstein, 1972; Davis 1983; Batson, Tricia, Highberger \& Shaw, 1995; Hoffman, 2000; Batson, Eklund, Chermok, Hoyt \& Ortiz, 2007). De acordo com Sampaio, Guimarães, Camino, Formiga e Menezes (2011; Formiga, Sampaio, Guimarães \& Camino, 2011) são muitos os estudos que buscam mensurar a empatia na ciência psicológica com forma, tipo e 
conceitualização de construto diversos. Essas mensurações buscam, conceitualmente, avaliar a capacidade do ser humano, em relação ao pensar, sentir e agir, na e com a experiência do outro, tendo como objetivo qualificar sua relação social e emocional.

Porém, é a escala de empatia de Davis (1983), conhecida no Brasil como Escala Multidimensional de Reatividade Interpessoal (EMRI), que possui um corpo teórico bem organizado, assim como, tem sido utilizada como variável explicativa em vários estudos no Brasil que salientam variáveis psicológicas e psicossociais (Camino \& Camino, 1996; Ribeiro, Koller \& Camino, 2002; Sampaio, Monte, Camino \& Roazzi, 2008; Formiga, Camino \& Galvao, 2009; Rique, Camino, Formiga \& Medeiros, 2010; Sampaio, Camino \& Roazzi, 2010).

A importância da escala desenvolvida por Davis para avaliar a empatia deve-se a perspectiva metodológica e teórica adotada por esse autor, na medida em que ele propõe um instrumento com construtos que condizem com uma visão psicogenética, evolutiva e multidimensional da empatia (Ribeiro, Koller \& Camino, 2002). De acordo com a perspectiva proposta por Davis (1983), as habilidades empáticas são distribuídas em quatro construtos independentes, os quais avaliam experiências afetivas e cognitivas da pessoa:

No que se refere à experiência cognitiva, destaca-se: O construto tomada de perspectiva do outro (refere-se à capacidade cognitiva voltada para a compreensão e coordenação de percepções do outro que visem à solução de conflitos interpessoais e sociais); e fantasia (refere-se a habilidade de se identificar com personagens ficcionais em novelas, filmes e romances e sentir junto com eles, uma adesão involuntária às condições afetivas de alegria, tristeza, raiva etc. e/ou de necessidade destes personagens).

Em relação à experiência afetiva: Esta poderá se acessada nas pessoas através da consideração empática (diz respeito à capacidade de avaliar e sentir com o outro, bem como do reconhecer seus afetos e necessidades, que pode ser experimentada no self como uma motivação de cunho pró-social que pode levar ao comportamento de ajuda) e a angústia pessoal (refere-se a um sentimento de tensão e desconforto, frente à condição de necessidade do outro, podendo gerar comportamentos de afastamento ao invés de comportamentos de ajuda).

Partindo dos pressupostos de que a orientação cultural influenciaria a empatia, realizou-se uma pesquisa nos sites de busca (Index Psi, 2011; Scielo, 2011) com as seguintes palavras-chave (Individualismo, coletivismo, empatia e 
Orientação cultual, empatia, jovens) não encontrando nenhum estudo que avaliasse as variáveis aqui abordadas. Com isso, neste estudo pretende-se, a partir da análise e modelagem de equação estrutural (MEE) através do programa AMOS 16.0, verificar empírica e teoricamente a associação entre o tipo de orientação cultural e a empatia em jovens brasileiros, bem como a qualidade e consistência explicativa entre as variáveis avaliadas.

O tipo de análise realizada neste estudo, vai além da análise de correlação clássica, pois aponta-se em direção de um inconveniente na segunda análise: a correlação pauta-se estritamente nos dados obtidos não considerando um modelo teórico fixo que oriente a extração das dimensões latentes e muito menos têm o poder de apresentar qualquer indicação sobre a bondade de ajuste do modelo. Estas técnicas - a da análise da modelagem estrutural - têm a clara vantagem de levar em conta a teoria para definir a associação entre as variáveis hipotetizadas, bem como, apresenta indicadores de bondade de ajuste que permitem decidir objetivamente sobre a validade de construto da medida analisada (Bilich; Silva \& Ramos, 2006; Hair; Anderson; Tatham \& Black, 2005).

Dois resultados principais podem ser esperados ao trabalhar com a análise estrutural: 1- estimativa da magnitude dos efeitos estabelecida entre variáveis, as quais estão condicionadas ao fato de o modelo especificado (isto é, o diagrama) estar correto, e 2 - testar se o modelo é consistente com os dados observados, a partir dos indicadores estatísticos, podendo dizer que resultado, modelo e dados são plausíveis, embora não se possa afirmar que este é correto (Farias \& Santos, 2000). Atende-se assim, não a certeza total do modelo, mas, a sua probabilidade sistemática na relação entre as variáveis.

Um dos principais objetivos das técnicas multivariadas - neste caso, considera-se a modelagem de equação estrutural - é expandir a habilidade exploratória do pesquisador e a eficiência estatística e teórica no momento em que se quer provar a hipótese levantada no estudo. Apesar das técnicas estatísticas tradicionais compartilharem de limitações, nas quais, é possível examinar somente uma relação entre as variáveis, é de suma importância para o pesquisador o fato de ter relações simultâneas; afinal, em alguns modelos existem variáveis que são independentes em algumas relações e, dependentes em outras (Silva, 2006; Hair, Anderson, Tatham \& Black, 2005; Hoe, 2008). De acordo com Farias e Santos (2000), Hair, Anderson, Tatham e Black (2005) e Zamora e Lemus (2008) ao considerar a modelagem estrutural do modelo - isto 
é, a análise de caminhos (path analysis) - relaciona-se as medidas de cada variável conceitual como confiáveis, acreditando que não existe erro de medida (mensuração) ou de especificação (operacionalização) das variáveis; cada medida é vista como exata manifestação da variável teórica.

Assim considerado, desenha-se o modelo teórico dos caminhos entre as variáveis independentes e dependentes que se pretende verificar a partir da hipótese elaborada; isto é, entre as variáveis latentes e variáveis observáveis, no desenho desse modelo, elabora-se a ligação entre as figuras caracterizando as variáveis estudadas - um retângulo é considerado como variável observada medida pelo pesquisador; uma elipse é considerada variável latente, isto é, construto hipotético não observado; uma seta com uma ponta indica o caminho ou a relação causal entre duas variáveis; uma seta com duas pontas representa a covariância, isto é, que estas variáveis se associam entre si; por fim, uma bolinha preenchida com um número e letra referem-se a um erro de medida. A partir do momento em que se elabora a hipótese, identifica cada uma dessas figuras associando as variáveis que se quer provar a múltipla influência.

Para que os resultados sejam obtidos faz-se necessário considerar índices de ajuste (escores co-variantes) - os quais, destacados na metodologia do presente estudo, na sessão do procedimento - permite enfatizar a teoria a que se propõem e sua explicação, simultaneamente, entre as variáveis independentes e dependentes, além de garantir uma melhor avaliação associativa entre as variáveis a que se pretende corroborar no modelo. A grande importância no uso dos estudos de modelagem de equação estrutural refere-se tanto em relação à segurança dos resultados multivariados, quanto, partindo de um estudo anterior ou de uma perspectiva teórica - ou até, de ambas - que avaliaram a associação simultânea entre as variáveis a serem testadas (Pitali \& Laros, 2007; Hoe, 2008).

Desta maneira, espera-se verificar as seguintes hipóteses:

H1 - o tipo de orientação cultural coletivista se associaria, positivamente, com a empatia;

H2 - por outro lado, o tipo de orientação cultural individualista se associaria, negativamente, com a empatia. 


\section{MÉTODO}

\section{Participantes}

Participaram do estudo 456 sujeitos, do sexo masculino (44\%) e do sexo feminino (56\%), de 12 a 67 anos $(M=24,62 ; D P=11,06)$. Estes foram do nível fundamental, médio e universitário de instituições privadas e de públicas das cidades de João Pessoa-PB e Rio de Janeiro-RJ. A amostra foi não probabilística, pois se considerou a pessoa que, consultada, se dispôs a colaborar, respondendo o questionário a ela apresentado.

\section{Instrumentos}

\section{Escala Multidimensional de Reatividade Interpessoal de Davis - EMRI}

Trata-se de um instrumento elaborado por Davis (1983) e adaptado em sua versão original por Sampaio, Guimarães, Camino, Formiga e Menezes (2011) para o contexto brasileiro. Um estudo posterior ao desses autores foi desenvolvido por Formiga, Sampaio, Guimarães e Camino (2011) verificando a acurácia do mesmo; observaram-se indicadores psicométricos $\left(\mathrm{X}^{2} / \mathrm{gl}=1,04\right.$; GFI=0,98; AGFI=0,96; RMR=0,03; $C F I=1,00 ; \operatorname{RMSEA}(90 \% \mathrm{IC})=0,00 \quad(0,00-$ 0,02); $C A I C=1208,51$ e ECVI=0,76) próximos aos encontrado por Sampaio e cols. (2011). O instrumento é composto por 26 sentenças que descrevem comportamentos, sentimentos e características relacionadas à empatia, que são utilizadas para avaliar as dimensões de experiência cognitiva, como a tomada de perspectiva do outro e a fantasia; e de experiência afetiva composta pela consideração empática e angústia pessoal. Todas foram avaliadas por escalas Likert, que variam de 1 ("não me descreve bem") a 5 ("descreve-me muito bem"). Escores mais altos indicam níveis mais elevados em cada uma dessas dimensões e a soma dos escores de todas as subescalas é utilizada para calcular o nível global de empatia.

Escala dos atributos do tipo de orientação cultural individualista e coletivista

Composto por seis itens que avaliam os atributos que mais caracterizam os sujeitos em relação ao individualismo e coletivismo (por exemplo, Um ser único, diferente dos demais; Orientado ao êxito, ao triunfo; Cooperador, que colabora; etc.). Formiga e Mota (2009) observaram Alfas de Cronbach aceitáveis pela literatura sobre o tema; realizando uma análise fatorial confirmatória (AFC) e a 
análise do modelo de equação estrutural (SEM), Formiga (2011) observou que este escala mostrou-se fidedigna quanto a estrutura fatorial já observada pelos autores supracitados, por exemplo: $x^{2} / g l=3,01 ;$ GFI=0,99 e AGFI=0,97; TLI=0,93; RMSEA $(90 \% \mathrm{IC})=0,05 \quad(0,03-0,08), \quad$ CAIC $=131,58$ e ECVI=0,07. Porém, no presente estudo considerou-se apenas quatro itens das facetas de individualismo (Ser único, diferente dos demais e Orientado ao êxito, ao triunfo) e coletivismo (Cooperador, que colabora e Cumpridor com os demais, ser servidor)

O instrumento proposto apresentou garantia de maior confiabilidade fatorial e evidências empíricas para sua aplicação e mensuração no contexto paraibano. Para respondê-lo o jovem deveria ler cada item e indicar o quanto cada um dos atributos lhe caracteriza, para isso, era necessário apontar (com um X ou circulo) numa escala do tipo Likert, com os seguintes extremos: $0=$ Nada Característico e $5=$ Muito Característico, ao lado dos respectivos atributos.

\section{Caracterização Sócio-Demográfica}

Foram elaboradas perguntas que contribuíram para caracterizar os participantes deste estudo (por exemplo, sexo, idade, classe sócio-econômica), bem como, realizar um controle estatístico de algum atributo que possa interferir diretamente nos seus resultados.

\section{Procedimentos}

Todos os procedimentos adotados nesta pesquisa seguiram as orientações previstas na Resolução 196/96 do CNS e na Resolução 016/2000 do Conselho Federal de Psicologia (CNS, 1996; ANPEPP, 2000).

\section{Administração}

Colaboradores com experiência prévia na administração da aplicação dos instrumentos foram responsabilizados pela coleta dos dados, e apresentaram-se nas salas de aula como interessados em conhecer as opiniões e os comportamentos dos alunos sobre as situações descritas nos instrumentos. Solicitou-se a colaboração voluntária dos jovens no sentido de responderem um breve questionário. Após ficarem cientes das condições de participação na pesquisa, assinaram um termo de Consentimento Livre e Esclarecido. Foi-Ihes dito que não havia resposta certa ou errada. A todos foi assegurado o anonimato 
das suas respostas informando que estas seriam tratadas em seu conjunto. As escalas foram então respondidas individualmente.

Apesar de o instrumento ser auto-aplicável, contando com as instruções necessárias para que possam ser respondidos, os colaboradores na aplicação estiveram presentes durante toda a aplicação para retirar eventuais dúvidas ou realizar esclarecimentos que se fizessem indispensáveis. Um tempo médio de 40 minutos foi suficiente para concluir essa atividade.

\section{Análise dos dados}

No que se refere à análise dos dados desta pesquisa, utilizou-se a versão 18.0 do pacote estatístico SPSS para Windows. Foram computadas estatísticas descritivas (tendência central e dispersão). Foram considerados os seguintes indicadores estatísticos para o Modelo de Equações Estruturais (MEE) segundo a forma de ajuste de erro.

Indicadores estatísticos para o Modelo de Equações Estruturais (SEM), efetuados no AMOS GRAFICS na versão 16.0, foram considerados segundo uma bondade de ajuste subjetiva:

- $O \mathrm{X}^{2}$ (qui-quadrado) testa a probabilidade de o modelo teórico se ajustar aos dados; quanto maior este valor pior o ajustamento. Este tem sido pouco empregado na literatura, sendo mais comum considerar sua razão em relação aos graus de liberdade ( $\mathrm{X}^{2} / \mathrm{g} . \mathrm{l}$.). Neste caso, valores até 5 indicam um ajustamento adequado (Joreskög \& Sörbom, 1989);

- Root Mean Square Residual (RMR), que indica o ajustamento do modelo teórico aos dados, na medida em que a diferença entre os dois se aproxima de zero. Para o modelo ser considerado bem ajustado, o valor deve ser menor que 0,05 (Bilich; Silva \& Ramos, 2006; Hair; Anderson; Tatham \& Black, 2005; Joreskög \& Sörbom, 1989);

- O Goodness-of-Fit Index (GFI) e o Adjusted Goodness-of-Fit Index (AGFI) são análogos ao $\mathrm{R}^{2}$ em regressão múltipla. Portanto, indicam a proporção de variância-covariância nos dados explicada pelo modelo. Estes variam de 0 a 1, com valores na casa dos 0,80 e 0,90, ou superior, indicando um ajustamento satisfatório (Hair, Anderson, Tatham \& Black, 2005; Bilich, Silva \& Ramos, 2006);

- O Root-Mean-Square Error of Approximation (RMSEA), com seu intervalo de confiança de $90 \%$ (IC90\%), considerando a estimação de resíduos a serem 
ajustados, isto é, valores altos indicam um modelo não ajustado. Assume-se como ideal que o RMSEA se situe entre 0,05 e 0,08, aceitando-se valores de até 0,10 (Kelloway, 1998; Garson, 2003);

- O Comparative Fit Index (CFI) - compara de forma geral o modelo estimado e o modelo nulo, considerando valores mais próximos de um como indicadores de ajustamento satisfatório (Joreskög \& Sörbom, 1989; Hair; Tatham; Anderson \& Black, 2005).

\section{RESULTADOS E DISCUSSÃO}

Pretende-se nesse estudo verificar a associação do tipo de orientação cultural sobre a empatia. Para isso, a partir da análise e modelagem de equação estrutural verificou-se, empíricamente o modelo hipotetizado. Considerou-se um modelo recursivo de equações estruturais efetuando-se o cálculo no programa AMOS 16.0. Na figura 1, realizadas as devidos ajustes de erro, encontrou-se um modelo adequado, para o tipo de orientação cultural coletivista e a empatia; este apresentou a seguinte razão: $x^{2} / g I=1,83 ; \mathrm{RMR}=0,02 ; \mathrm{GFI}=0,99 ; \mathrm{AGFI}=0,97$; $\mathrm{CFI}=1,00 ; \mathrm{RMSEA}=0,04(0,00-0,08)$. Observou-se que 0 peso da variável considerada - tipo de orientação coletivista - apresentou um escore associativo positivo $(\Lambda=0,41)$ em relação à empatia.

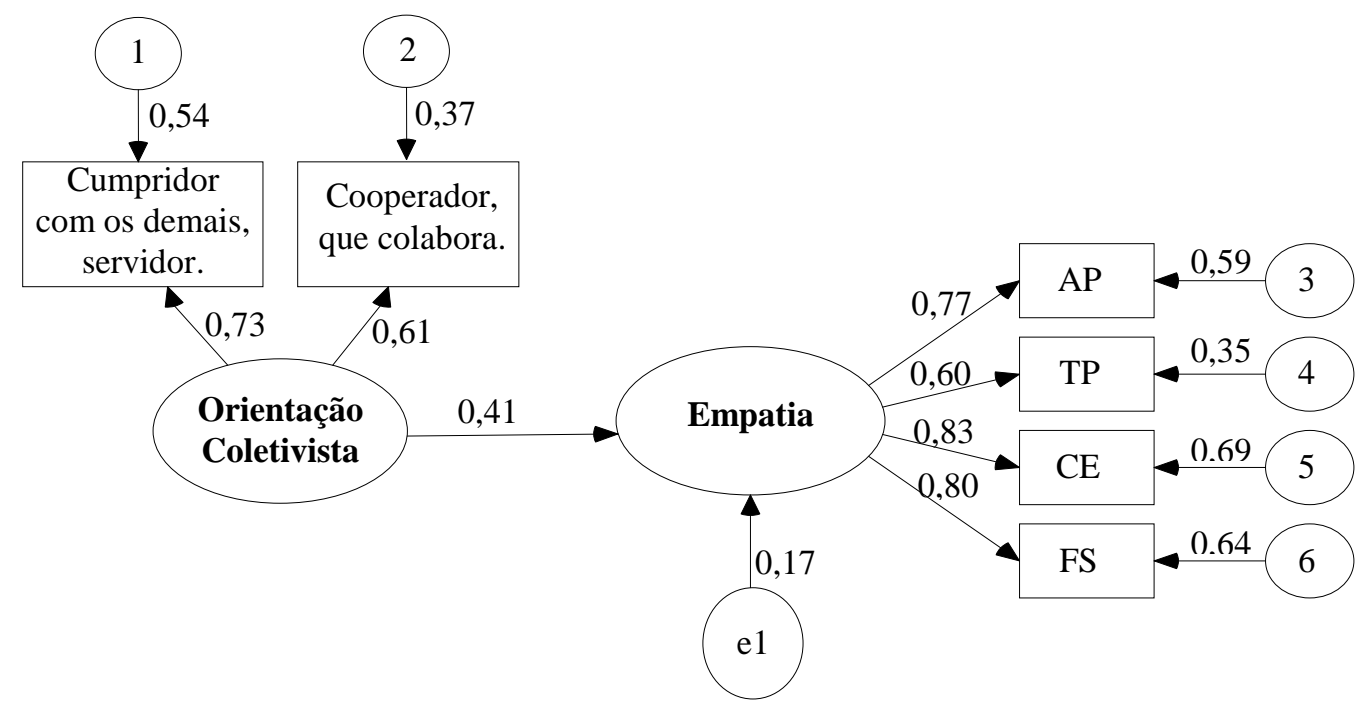

Figura 1. Modelo teórico para explicação da orientação coletivista sobre a empatia. Nota: Consideração Empática (CE), Angústia Pessoal (AP) e Tomada de Perspectiva (TP) e Fantasia (FS). 
Em relação à influência do tipo de orientação cultural individualista e a empatia, o mesmo procedimento realizado para o teste do primeiro modelo foi efetuado. Desta forma, na figura 2, feitas as devidas modificações nos ajustes de erro, encontrou-se um modelo adequado, no qual o tipo de orientação cultural individualista se associou, negativamente $(\Lambda=-0,19)$, a empatia; tendo os seguintes indiciadores: $x^{2} / g l(3,59 / 7)=0,51 ; R M R=0,01 ; G F I=1,00 ; A G F I=0,99$; $\mathrm{CFI}=1,00 ; \mathrm{RMSEA}=0,00(0,00-0,03)$.

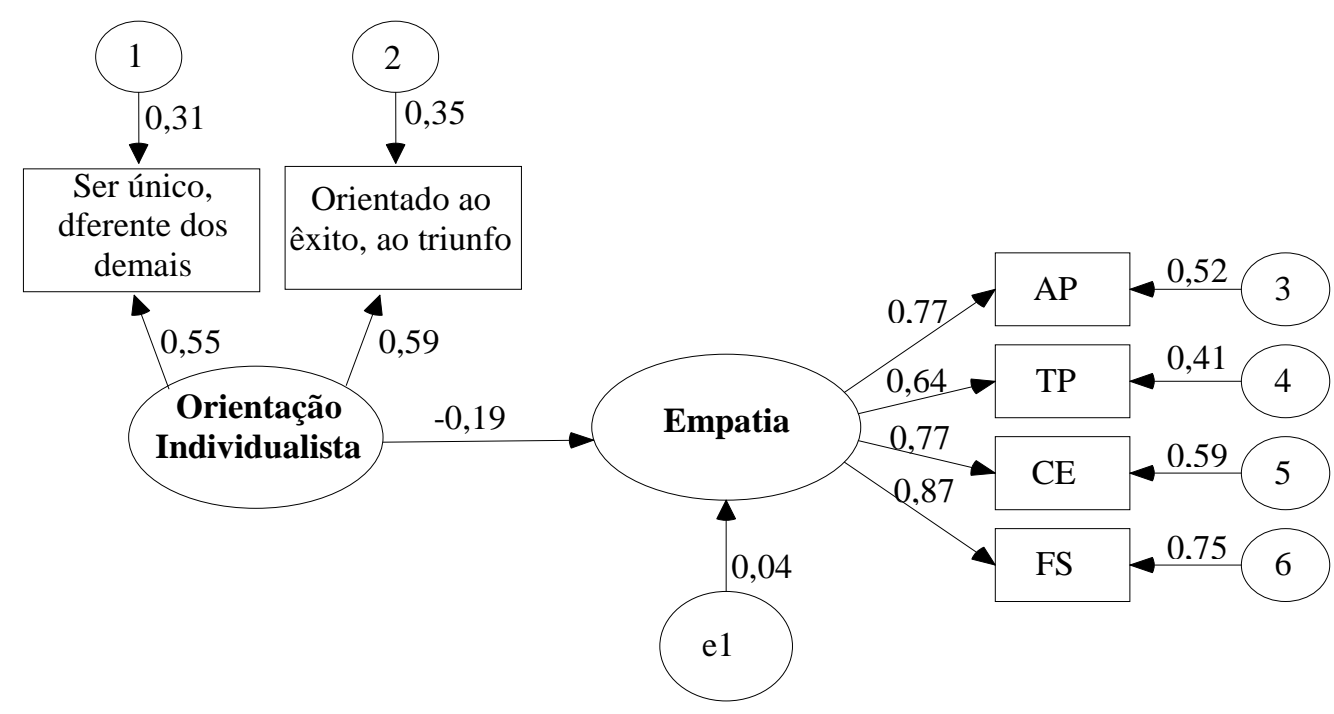

Figura 2. Modelo teórico para explicação da orientação individualista sobre a empatia. Nota: Consideração Empática (CE), Angústia Pessoal (AP) e Tomada de Perspectiva (TP) e Fantasia (FS).

Considerando que os modelos teóricos propostos foram comprovados, pois apresentaram indicadores psicométricos para a sua estrutura, aceito na literatura estatística (Joreskög \& Sörbom, 1989; Hair, Anderson, Tatham \& Black, 2005), corroborando a associação entre o tipo de orientação cultural e a empatia: por um lado, a orientação coletivista associou-se positivamente com a empatia, por outro lado, a orientação individualista a fez negativamente. Desta forma, optouse em realizar uma Manova a fim de avaliar as diferenças nas pontuações médias nas variáveis do estudo; por exemplo, Estado (município) x orientação cultura x empatia. Para isso, duas etapas foram realizadas, efetuou-se uma análise multivariada e estabeleceu, para o construto da empatia a avaliação desta, a partir da experiência afetiva e cognitiva da empatia. Tomou-se com base para a 
construção dessa variável o somatório dos componentes empáticos - compostos pelas dimensões empáticas $C E, T P, A P$ e FS - os quais referem-se a cada experiência empática, já estabelecidos por Davis (1983).

\section{Variação na orientação cultural coletivista e a experiência empática}

Tendo como variável dependente o Estado (Rio de Janeiro e João Pessoa) e o tipo de orientação cultural coletivista (baixo e alta orientação) versus as experiências empáticas (afetiva e cognitiva) observaram-se os seguintes resultados:

- houve resultado significativo no efeito principal na variável Estado em relação à experiência afetiva da empatia; os sujeitos do Rio de Janeiro-RJ pontuaram mais alto $(M=3,86, D P=0,046 ; I C-3,76-3,95)$ do que os de João Pessoa-PB ( $M=3,60$, DP=0,050; IC95\% - 3,50-3,70) $[F(1,486)=14,21 ; p<0,01]$;

- no que se refere à experiência cognitiva da empatia, observou-se, também, que na amostra do Rio de Janeiro-RJ, os sujeitos apresentaram medias superiores ( $M=3,51, D P=0,04 ;$ IC $-3,44-3,58)$ quando comparado a amostra de João Pessoa-PB (M=3,35, DP=0,03; IC95\% - 3,27-3,43) $\quad[F(1,486)=9,25$; $p<0,01]$. Encontrou-se, para esses resultados multivariados, um valor $F$ de 7,15 , com um Lambda $(\square)$ Wilks de 0,97 .

- resultados significativos foram encontrados, também, no efeito principal, para variável tipo de orientação cultural coletivista em relação à experiência afetiva da empatia; os sujeitos apresentaram médias superiores no alto coletivismo $(M=3,89, D P=0,06$; IC $-3,78-4,00)$ do que no baixo coletivismo $(M=3,56, D P=0,04 ; I C 95 \%-3,48-3,64)[F(1,486)=22,82 ; p<0,01]$;

- em relação à experiência cognitiva da empatia, os sujeitos tiveram médias superiores na alta orientação coletivista $(M=3,57, D P=0,04$; IC $-3,48-3,65)$ do que no baixo coletivismo ( $M=3,30, \quad D P=0,03 ; \quad I C 95 \% \quad-3,24-3,36)$ $[F(1,486)=25,56 ; p<0,01] ;$ para esses resultados multivariados, observou-se um valor $\mathrm{F}$ de 13,91 , com um Lambda $(\Lambda)$ Wilks de 0,95 .

Variação na orientação cultural individualista e a experiência empática

Considerando, ainda, a variável dependente o Estado (Rio de Janeiro e João Pessoa) e o tipo de orientação cultural individualista (baixo e alta orientação) em relação às experiências empáticas (afetiva e cognitiva), observaram-se resultados significativos apenas para o efeito principal na variável Estado: 
- em relação à experiência afetiva da empatia, os sujeitos do Rio de Janeiro-RJ tiveram médias superiores $(M=3,77, D P=0,04 ;$ IC $-3,68-3,85)$ do que os de João Pessoa-PB (M=3,59, DP=0,05; IC95\% - 3,49-3,69) $[F(1,486)=6,86$; $p<0,01]$

- no que diz respeito à experiência cognitiva da empatia, também, os sujeitos do Rio de Janeiro-RJ tiveram médias superiores $(M=3,45, D P=0,03$; IC 3,39-3,52) do que os sujeitos de João Pessoa-PB ( $M=3,34$, DP=0,04; IC95\% 3,26-3,42) $\quad[F(1,486)=4,20 ; p<0,05]$; encontrou-se, para esses resultados multivariados, um valor $\mathrm{F}$ de 3,42, com um Lambda $(\Lambda)$ Wilks de 0,99.

A partir desses resultados, não somente se corroborou a hipótese levantada em relação da influência do tipo de orientação cultural sobre a empatia, especificamente, na existência de uma variação na orientação coletivista ou individualista, as quais, provavelmente, interfeririam na experiência - afetiva e cognitiva - da empatia. Vale destacar, também, que o contexto sócio-cultural (considerando os Estados em que foram realizadas as pesquisas), também, contribuíram para avaliar a maior intensidade na experiência empática e na orientação cultural. Neste caso, quanto maior a orientação cultural coletivista maior a experiência empática afetiva e cognitiva.

Em ambas as experiências, de acordo com Sampaio, Guimarães, Camino, Formiga e Menezes (2011) e Formiga, Sampaio, Guimarães e Camino (2011), as pessoas seriam capazes de, tanto pensarem (compreendendo e organizando suas percepções a respeito do outro) em como inibir ou diminuir o conflito interpessoal, quanto desenvolver uma capacidade de avaliação e do sentimento em relação ao outro. Tais condições seriam úteis ao enfatizar os contextos sociais vividos pelo sujeito, principalmente, quando nestes contextos forem incluídas propostas e intervenções dos órgãos públicos na prevenção e inibição de atitudes de riscos e envolvimento cooperativo da população, seja em relação à violência, ao apoio social e material quando em tragédias ou situação conflitante de reinvindicação de estados de direitos. Isto é, pensa e sentir a situação em que o outro possa estar envolvido exige tanto um desenvolvimento psicológico quanto a orientação cultural - de individualismo ou coletivismo - a qual a pessoa está sendo socializada.

Com isso, a importância do tipo de orientação cultural coletivista, bem como, das experiências empáticas, podem ser destacadas devido à possibilidade de se desenvolver no sujeito um melhor deslocamento de ego, já que em ambos 
os construtos exige-se que a pessoa saia de si para atender o outro ou para diminuir seu conflito, tensão ou desconforto pessoal. Afinal, a orientação coletivista horizontal (cooperador, que colabora) ou vertical (cumpridor com os demais, ser servidor) estimula o sujeito na busca da harmonia e respeito às tradições e hierarquia social visando à melhoria de todos (Hofstede, 1980; Triandis, 1995), condição esta, que possivelmente poderia ser atribuído ao desenvolvimento das habilidades empáticas nas pessoas (Davis, 1983), a qual fomentaria na pessoa tanto uma melhoria nas relações humanas quanto uma racionalização sobre suas atitudes em relação ao outro e seus investimentos prósociais.

Investir em uma orientação coletivista, provavelmente, contribuiria para a compreensão das pessoas sobre sua capacidade de abrir canais comunicativos para a relação interpessoal quanto ao estimulo e simulação de convicções, desejos, percepções sobre o contexto do outro, observando os sentimentos e as emoções que o conduziriam para ser empático. Ao trabalhar com a empatia, a partir das orientações culturais, esta, teria a função de gerar no sujeito um compromisso que permita predizer o comportamento do outro frente as atitudes, crenças, definições do self, etc., na valoração e importância do outro, promovendo com isso uma agenda de pesquisa psicossocial a respeito de mais um fator de proteção da vulnerabilidade social e psíquica, bem como, para uma organização e manutenção da condição de resiliência frente às adversidades interpessoais na dinâmica da sociedade contemporânea (Eisenberg \& Strayer, 1990).

Considerando a reflexão do parágrafo acima, é possível destacar a diferença existente nos resultados encontrado na amostra do Rio de Janeiro-RJ e João Pessoa-PB; é destaque que na amostra do Rio de Janeiro a pontuação média das respostas dos sujeitos foi maior no construto da empatia e na orientação coletivista, do que a pontuação dos sujeitos da amostra de João Pessoa. Essa condição, provavelmente, se deve aos novos investimentos sociais e humanos promovido pelo Estado e organizações leigas e comunidade em geral em relação aos inúmeros acontecimentos de risco, desvios sociais e crimes, ocorridos nos últimos dois anos neste Estado. Estes investimentos mobilizaram-se atividades contra a violência, valorização pela vida e paz e colaboração com as pessoas que perderam, em tragédias naturais, entes queridos e posses materiais. 
Por fim, espera-se que os objetivos deste estudo tenham sido cumpridos, principalmente, no que diz respeito comprovação do modelo causal entre o tipo de orientação cultural e a empatia, empregando em áreas de estudo que cooperam com a psicologia, educação, sociologia, etc. Apesar dos resultados serem satisfatórios, faz-se necessário ter em conta aspectos mais específicos ou universais de cada, quando se pretender avaliar essas variáveis em outras culturas (Muenjohn \& Armstrong, 2007; Triandis e cols., 1993; Triandis, 1994; Van De Vijver \& Leung, 1997).

Desta maneira, aponta-se para a seguinte direção: conhecer os aspectos que podem ser comuns a todas as culturas e aqueles que são específicos, contribuindo para consolidar um marco teórico do tipo de orientação cultural e da empatia, já que, considerando os resultados encontrados é possível que ambas as variáveis podem se diferenciar entre si devido aos contextos sociais e políticos. Com isso, o que fazer no futuro em relação a essas escalas? Pretendese reunir evidências adicionais da consistência intra, inter e pan-culturais em relação a estes medidas e o modelo teórico, bem como, a realização estudos que avaliasse além das experiências empáticas, as dimensões da empatia da tomada de perspectiva do outro, fantasia, consideração empática e angustia Pessoal e sua associação com as orientações culturais; outra condição importante para futuros estudos refere-se, relacionado ao conjunto dessas variáveis já destacadas neste parágrafo, a uma análise controlando o gênero e a idade.

\section{REFERÊNCIAS}

Associação Nacional de Pesquisa e Pós-graduação em Psicologia - ANPEPP (2011). Contribuições para a discussão das Resoluções CNS no. 196/96 e CFP No 016/2000. Recuperado em 02 de Setembro de 2011, da WEB (página da WEB): http://www.anpepp.org.br/XIISimposio/Rel_ComissaoEticasobre_Res_CNS_ e_CFP.pdf2000.

Atteslander, P. (1999). Social Change, Development and Anomie. In: Peter Atteslander, Bettina Gransow \& John Western (Org). Comparative anomie research: Hidden barriers - hidden potential for social development. Sidney: Ashgate.

Barzun, J. (2002). Da alvorada à decadência: A história da cultura ocidental de 1500 aos nossos dias. Rio de Janeiro: Campus. 
Batson, C. D.; Eklund, J. H.; Chermok, V. L.; Hoyt, J. L. \& Ortiz, B. G. (2007). An additional antecedent of empathic concern: valuing the welfare of the person in need. Journal of Personality and Social Psychology, 93(1), 65-74.

Batson, D. C.; Tricia, R. K.; Highberger, L. \& Shaw, L. L. (1995). Immorality From Empathy-Induced Altruism: When Compassion and Justice Conflict. Journal of Personality and Social Psychology, 68(6), 1042-1054.

Bauman, Z. (1998). O mal-estar da pós-modernidade. 1.ed. Rio de Janeiro: Jorge Zahar Ed.

Bilich, F.; Silva, R.; Ramos, P. (2006). Análise de flexibilidade em economia da informação: Modelagem de equações estruturais. Revista de Gestão da Tecnologia e Sistemas de Informação, 3(2), 93-122.

Bürger, P. (1988). O declínio da era moderna. Revista Novos Estudos CEBRAP, 20, 81-95.

Byrne, B. M. (1989). A primer of LISREL: Basic applications and programming for confirmatory factor analytic models. New York: Springer-Verlag.

Camino, C. \& Camino, L. (1996). Julgamento moral, emoção e empatia. In Z. D. Trindade \& C. Camino (Eds.), Cognição social e juízo moral (Coletâneas da ANPEPP). (pp. 109-135). Rio de Janeiro: Associação Nacional de Pesquisa e Pós-graduação em Psicologia.

Conselho Nacional de Saúde - CNS. (1996). Diretrizes e Normas Regulamentadoras de Pesquisas Envolvendo Seres Humanos. Recuperado em 02 de Setembro de 2011, da WEB (página da WEB): http://conselho.saude.gov.br/resolucoes/reso_96.htm.

Davis, M. H. (1983). Measuring individual differences in empathy: Evidence for a multidimensional approach. Journal of Personality and Social Psychology, $44,113-126$.

Decety J.; Michalska K. J. \& Akitsuki, Y. (2008). Who caused the pain? A functional MRI investigation of empathy and intentionality in children. Neuropsychologia, 46, 2607-2614.

Decety, J. \& Jackson, P. L. (2004). The functional architecture of human empathy. Behavioral and Cognitive Neuroscience Reviews, 3, 71-100.

Decety, J. (2005). Perspective taking as the royal avenue to empathy. In B. F. Malle e S. D. Hodges (Eds.), Other minds: How humans bridge the divide between self and other. (pp. 143-157). New York: Guilford Publications. 
Depraz, Natalie. A ética relacional: Uma prática de ressonância interpessoal. Revista do departamento de Psicologia da UFF, 17(2), 19-34.

Dumont, L. (1985). O individualismo: Uma perspectiva antropológica da ideología moderna. Rio de Janeiro: Rocco.

Eisenberg, N. \& Strayer, J. (1990). Empathy and its development. New York: Cambridge University Press.

Elias, N. (1994). O processo civilizador: Formação do Estado e Civilização. Rio de Janeiro: Jorge Zahar. Vol. 2

Enz, N. \& Zoll, N. (2006). Cultural differences in empathy between China, Germany and the UK. Recuperado em 23 de novembro de 2006, de www.nicve.salford.ac.uk/elvis/ resources/empathy.

Farias, S. A. \& Santos, R. C. (2000). Modelagem de Equações Estruturais e Satisfação do Consumidor: uma Investigação Teórica e Prática. Revista de Administração Contemporânea, 4(3), 107-132.

Fernández-Armesto, F. (1999). Milênio: Uma história de nossos últimos mil anos. Rio de Janeiro: Record.

Formiga, N. S. \& Diniz, A. (2011). Estilo da orientação cultural e condutas desviantes: Testagem de um modelo teórico. Revista Pesquisa em Psicologia, 5(1), 2-11.

Formiga, N. S. \& Mota, H. M. (2009). Estilo da orientação cultural e condutas desviantes: Um estudo correlacional em jovens paraibanos. Cadernos de Pesquisa Interdisciplinar em Ciências Humanas, 10(97), 158-180

Formiga, N. S. (2004). O tipo de orientação cultural e sua influência sobre os indicadores do rendimento escolar. Psicologia: Teoria e Prática, 16(1), 1329.

Formiga, N. S.; Camino, C. \& Galvão, L. (2009). Empatia, desenvolvimento moral e conduta desviante em adolescentes: testagem de um modelo teórico. In: VII Congresso Brasileiro de Psicologia do Desenvolvimento, 2009, Rio de Janeiro. (pp. 541-542). Rio de Janeiro, RJ: CBPD.

Formiga, N. S.; Sampaio, L. R.; Guimaraes, P. R. B. \& Camino, C. P. S (2011). Escala multidimensional de reatividade interpessoal de Davis: Quatro ou três fatores mensuram a empatia? Artigo submetido à avaliação.

Garson, G. D. (2003). PA 765 Statnotes: An online textbook. Endereço de página Web: http://www2.chass.ncsu.edu/garson/pa765/statnote.htm (consultado dia 17 de maio de 2005). 
Gouveia, V. V.; Clemente, M. \& Vidal, M.A. (1998). España desde dentro: el individualismo y el colectivismo como rasgos diferenciadores de las comunidades autónomas. Sociedade y Utopia, 11, 168-179.

Hair, J. F.; Anderson, R. E.; Tatham, R. L.; Black, W. (2005). Análise multivariada de dados. Porto Alegre: Bookman.

Hoe, L. S. (2008). Issues and procedures in adopting structural equation modeling technique. Journal of Applied Quantitative Methods, 3(1), 76-83.

Hoffman, M. L. (2000). Empathy and moral development: Implications for caring and justice. New York: Cambridge University Press.

Hofstede, G. (1980). Culture's consequences. Beverly Hills, CA: Sage.

Howard, G. S. (2000). Adapting human lifestyles for the 21st century. American Psychologist, 55(5), 509-515,

Index psi. (2011). Orientação cultual, empatia, jovens (Pagina da web: http://www.scielo.br. Pesquisa realizada em 05 de Dezembro de 2011).

Inglehart, R. (1991). El cambio cultural en las sociedades industriales avanzadas. Madrid: Centro de Investigaciones Sociológicas / Siglo XXI Editores.

Jodelet, D. (1984). Représentation sociale: phénomènes, concept et théorie. In: S. Moscovici (Ed.) Psychologie Sociale (pp. 357-378). Paris: Presses Universitaires de France.

Joreskög, K. \& Sörbom, D. (1989). LISREL 7 user's reference guide. Mooresville: Scientific Software.

Kelloway, E. K. (1998). Using LISREL for structural equation modeling: $A$ researcher's guide. Thousand Oaks, CA: Sage Publications.

Kumar, K. (1997). Da sociedade pós-industrial à pós-moderna: Novas teorias sobre o mundo contemporâneo. Rio de Janeiro: Zahar.

Lipovetsky, G. \& Charles, S. (2004). Os tempos hipermodernos. São Paulo: Barcarolla,

Lipovetsky, G. (1986). La era del vacío: Ensayos sobre el individualismo contemporáneo. Barcelona: Editorial Anagrama.

Lyotard, J. -F. (2000). A condição pós-moderna. Trad. Ricardo Corrêa Barbosa. $6^{\mathrm{a}}$ ed. Rio de Janeiro: José Olympio.

Mehrabian, A. \& Epstein, N. (1972). A measure of emotional empathy. Journal of Personality, 40, 525-543. 
Muenjohn, N. \& Armstrong, A. (2007). Transformational leadership: The influence of culture on the leadership behaviours of expatriate managers. International Journal of Business and Information, 2(2), 265-283.

Pilati, R. \& Laros, J. A. (2007). Modelos de equações estruturais em Psicologia: Conceitos e aplicações. Psicologia: Teoria e Pesquisa, 23(2), 205-216.

Ribeiro, J.; Koller, S. H. \&Camino, C. (2002). Adaptação e validação de duas escalas de empatia para uso no Brasil. Estudos de Psicologia, 18(3), 43-53.

Rique, J.; Camino, C.; Formiga, N. S. \& Medeiros, F. (2010). Consideração empática e tomada de perspectiva para o perdão interpessoal. Interamerican Journal of Psychology, 44, 515-522,

Rokeach, M. (1973). The nature of human values. New York: The Free Press.

Rokeach, M. (1979). Introduction. Em M. Rokeach (Ed.), Understanding human values: Individual and societal. (pp. 1-11). New York: The Free Press.

Sampaio, L. R.; Camino, C. P. S. \& Roazzi, A. (2009). Revisão de aspectos conceituais, teóricos e metodológicos da empatia. Psicologia: Ciência e profissão, 29(2), 212-227.

Sampaio, L. R.; Guimarães, P. R. B.; Camino, C. P. S; Formiga, N. S. \& Menezes, I. G. (2011). Estudos sobre a dimensionalidade da empatia: tradução e adaptação do Interpersonal Reactivity Index (IRI). Psico, 42(1), 67-76.

Sampaio, L. R.; Monte, F. C.; Camino, C. \&Roazzi, A. (2008). Justiça distributiva e empatia em adolescentes do nordeste brasileiro. Psicologia: Reflexão e Crítica, 21(2), 275-282.

Schwartz, S. H. (1990). Individualism-collectivism: Critique and proposed refinements. Journal of Cross-Cultural Psychology, 21, 139-157.

Scielo. (2011). Individualismo, coletivismo, empatia. (Pagina da web: http://www.scielo.br. Pesquisa realizada em 20 de Novembro de 2011).

Silva, J. S. F. (2006). Modelagem de equações estruturais: Apresentação de uma metodologia. Dissertação (Mestrado em Engenharia de Produção). Universidade Federal do Rio Grande do Sul. Recuperado em 25 de agosto de 2009, da WEB (página da WEB): http://hdl.handle.net/10183/8628.

Sinha, D. \& Tripathi, R. C. (1994). Individualism in a collectivist culture: A case of coexistence of opposites. Em U. Kim, H.C. Triandis, Ç. Kagitçibasi, S.-C. Choi \& G. Yoon (Eds.), Individualism and collectivism: Theory, method, and applications. (pp. 123-136). Thousand Oaks, CA: Sage Publications. 
Souza, M. A. (2003). Representação social da sociedade, anomia e individualismo-coletivismo. Tese de Doutorado não publicada. Universidade Federal do Rio de Janeiro - UFRJ, Instituto de Psicologia.

Teixeira, E. B. (2005). Aventura pós-moderna e sua sombra. São Paulo-SP: Paulus.

Triandis, H. C. (1995). Individualism and collectivism. Boulder, CO: Westview Press.

Triandis, H. C. (1996). The psychological measurement of cultural syndromes. American Psychologist, 51, 407-415.

Triandis, H.C., Chen, X.P. \& Chan, D. K. S. (1998). Scenarios for the measurement of collectivism and individualism. Journal of Cross-Cultural Psychology, 29, 275-289.

Trianis, H. C.; Mccusker, C.; Betancourt, H.; Iwao, S.; Leung, K.; Salazar, J. M. ; Setiadi, B.; Sinha, B. P.; Touzard, H.; Zaleski, Z. (1993). An etic-emic analysis of individualism and collectivism. Journal of cross-cultural psychology, 24(3), 366-383.

Van De Vijver, F. \& Leung, K. (1997). Methods and data analysis for crosscultural research. Thousand Oaks, CA: Sage Publications.

Wispé, L. (1990). History of the concept of empathy. In: N. Eisenberg \& J. Strayer (org), Empathy and its development. (pp 17-37). New York: Cambridge University Press.

Contato: nsformiga@yahoo.com, maguiarsouza@uol.com.br

Recebido em: 25/07/2012

Revisado em: 24/11/2012

Aceito em: 27/12/2012 